\title{
Effects of multidisciplinary team working on breast cancer survival: retrospective, comparative, interventional cohort study of 13722 women
}

\author{
(C) $(1) \Theta$ OPEN ACCESS
}

\author{
Eileen M Kesson project manager ${ }^{14}$, Gwen M Allardice statistician ${ }^{14}$, W David George school of \\ medicine honorary professor ${ }^{2}$, Harry J G Burns chief medical officer for Scotland ${ }^{3}$, David S Morrison \\ director $^{4}$
}

${ }^{1} \mathrm{NHS}$ Greater Glasgow and Clyde, West House, Gartnavel Royal Hospital, Glasgow, UK; ${ }^{2}$ Western Infirmary, Glasgow; ${ }^{3}$ Scottish Government, Public Health Division, Edinburgh, UK; ${ }^{4}$ West of Scotland Cancer Surveillance Unit, Institute of Health and Wellbeing, University of Glasgow, Glasgow G12 8RZ

\begin{abstract}
Objectives To describe the effect of multidisciplinary care on survival in women treated for breast cancer.

Design Retrospective, comparative, non-randomised, interventional cohort study.

Setting NHS hospitals, health boards in the west of Scotland, UK.

Participants 14358 patients diagnosed with symptomatic invasive breast cancer between 1990 and 2000, residing in health board areas in the west of Scotland. 13722 (95.6\%) patients were eligible (excluding 16 diagnoses of inflammatory cancers and 620 diagnoses of breast cancer at death).

Intervention In 1995, multidisciplinary team working was introduced in hospitals throughout one health board area (Greater Glasgow; intervention area), but not in other health board areas in the west of Scotland (non-intervention area).
\end{abstract}

Main outcome measures Breast cancer specific mortality and all cause mortality.

Results Before the introduction of multidisciplinary care (analysed time period January 1990 to September 1995), breast cancer mortality was $11 \%$ higher in the intervention area than in the non-intervention area (hazard ratio adjusted for year of incidence, age at diagnosis, and deprivation, $1.11 ; 95 \%$ confidence interval 1.00 to 1.20 ). After multidisciplinary care was introduced (time period October 1995 to December 2000), breast cancer mortality was $18 \%$ lower in the intervention area than in the non-intervention area $(0.82,0.74$ to 0.91$)$. All cause mortality did not differ significantly between populations in the earlier period, but was $11 \%$ lower in the intervention area than in the non-interventional area in the later period $(0.89,0.82$ to 0.97$)$. Interrupted time series analyses showed a significant improvement in breast cancer survival in the intervention area in 1996, compared with the expected survival in the same year had the pre-intervention trend continued
$(P=0.004)$. This improvement was maintained after the intervention was introduced.

Conclusion Introduction of multidisciplinary care was associated with improved survival and reduced variation in survival among hospitals. Further analysis of clinical audit data for multidisciplinary care could identify which aspects of care are most associated with survival benefits.

\section{Introduction}

Breast cancer is the commonest cancer in women worldwide. ${ }^{1}$ Its incidence has increased over several decades, while the death rate has fallen because of improved survival. ${ }^{23}$ Survival has probably improved because of a mixture of earlier detection of the disease (both through screening and earlier symptomatic presentation $),{ }^{4}$ biological changes that have made the disease more susceptible to hormonal therapy, ${ }^{5}{ }^{6}$ and improved treatment. $^{7}$

Treatment of cancer has increasingly been provided within centralised, specialist multidisciplinary teams in Europe, the United States, and Australia. ${ }^{8-12}$ These teams were established after observational evidence identified better outcomes among patients treated by specialists for various common cancers ${ }^{13}$, such as an $11-17 \%$ reduction in risk of death among women treated for breast cancer by specialist surgeons ${ }^{14}$ However, it remains unclear whether multidisciplinary care improves cancer survival and whether the costs justify clinical benefits. ${ }^{15-18}$ Although attempts have been made to estimate the costs of multidisciplinary teams, ${ }^{15}$ we are not aware of comparisons with costs for conventional care.

Although some evidence has shown improved outcomes in cancer associated with the introduction of multidisciplinary care, ${ }^{19-21}$ a recent systematic review of 21 studies (including five on breast cancer) could not determine a causal relation between 
such care and cancer survival for three main reasons. ${ }^{18}$ Firstly, the definition of multidisciplinary care was imprecise and heterogeneous. Secondly, the outcomes of multidisciplinary care could have indicated selection biases towards patients with more favourable prognostic features rather than true treatment effects. Thirdly, no adjustment was made for the confounding effects of temporal improvements in survival that might have occurred without the introduction of multidisciplinary care. For example, none of the identified studies included a contemporaneous comparison group. ${ }^{18}$

In the early 1990s, variations in survival among hospitals managed by the Greater Glasgow health board were reported to the director of public health (HJGB). The hospital with highest survival had delivered care in a multidisciplinary manner. As a result, multidisciplinary teams were formally established in October 1995 throughout the Greater Glasgow health board area, with the aim of improving survival and minimising variations among hospitals. No substantial reorganisation of breast cancer care took place in areas managed by other health boards in the west of Scotland until 2000, when national guidance was introduced. ${ }^{22}$

We aimed to determine whether the introduction of multidisciplinary care affected the survival of women with breast cancer. Multidisciplinary teams were introduced to Greater Glasgow health board as an NHS Scotland area-wide policy to improve the quality of care. Therefore, an experimental study design, such as the randomisation of patients to hospitals providing or not providing multidisciplinary care, would not be possible. We compared an intervention area that had introduced multidisciplinary care with neighbouring areas (without such care), before and after the organisational change. We addressed the limitations of previous studies,${ }^{18}$ by defining the composition of a multidisciplinary team at the outset, observing the effect of care where no other model was available, and including a contemporaneous comparison population to adjust for temporal improvements in survival.

\section{Methods}

\section{Design and patients}

We did a retrospective, comparative, non-randomised, interventional cohort study. From the Scottish Cancer Registry, we selected data for all incident, symptomatic invasive breast cancers (ICD-10, international classification of diseases (ICD), 10th revision, codes C50.1 to C50.9) diagnosed between 1 January 1990 and 31 December 2000 for residents of health board areas in the west of Scotland. We obtained data from the Scottish Cancer Registry, which is a comprehensive account of all incident cancers gathered from pathology, hospital, and death records, among other sources. The registry is linked, by probability matching, to death records from the General Register Office for Scotland and has a high level of accuracy. ${ }^{23}{ }^{24}$ Patients were grouped according to the health board of diagnosis. The Greater Glasgow health board area (population 900 000) was the intervention area, and areas managed by the remaining health boards in the west of Scotland (1 600000$)$ comprised the non-intervention area.

Data for tumour size was only available in the Scottish Cancer Registry from 1997 onwards. If data were available, we grouped tumour size. ${ }^{14}$ We calculated age at diagnosis for all patients, and used four age groups: younger than 50 years, which is considered to be a proxy for premenopausal status ${ }^{25} ; 50-64$ years, the age band at which patients would be invited for breast screening; 65-79 years, elderly and post screening age; and 80 years and older. The oldest age group has been shown to have survival equal to that of younger women after treatment of breast cancer $^{26}$; the most appropriate management in this age group also remains unclear. ${ }^{27}$

We included patients if they were diagnosed with breast cancer but had no other primary cancers, were resident in and attending an NHS hospital managed by one of the health boards in the west of Scotland, and were not diagnosed by the National Breast Screening Programme. Tumours detected by screening programmes are usually at earlier stages with better prognoses. Although screen detected patients might have been diagnosed by the programme throughout the west of Scotland, they were treated mainly in two hospitals managed by the Greater Glasgow health board.

\section{Multidisciplinary team intervention}

In 1995, multidisciplinary team care for all patients with breast cancer was introduced in the Greater Glasgow health board area (intervention area), but not in the areas managed by other health boards in the west of Scotland (non-intervention area). Five multidisciplinary teams were formed in the intervention area, each led by a specialist surgeon performing in excess of 50 operations for invasive breast cancer each year. In 1995, a multidisciplinary team was defined as a group with the following characteristics:

- Comprised specialist breast cancer surgeons, pathologists, oncologists, radiologists, and specialist nurses

- Worked to evidence based guidelines, written by the specialist breast surgeon whose patients had the highest survival rates before reorganisation of care delivery (WDG)

- Held weekly formal meetings to discuss results and agree on adjuvant treatment for individual patients

- Audited clinical activity and reported results at regular intervals

- Lead clinicians from each team met regularly with the director of public health (HJGB) to discuss audit results throughout the area, with the aim to minimise deviations from guidelines and variations in practice and improve quality of care.

Doctors operating on small numbers outside the five main centres were no longer allowed to manage patients needing care for breast cancer. As a result, there was a degree of centralisation of services. However, the geographical location of the hospitals in the intervention area was such that patients did not have to travel long distances to receive care.

No formal change in delivery of care was introduced in the non-intervention area; patients continued to receive care along more traditional approaches, with surgeons making unilateral decisions regarding surgery and adjuvant treatment. In 1999, Scottish guidance was issued on the reorganisation of cancer care. ${ }^{22}$ In 2001-02, services throughout the country began to form managed clinical networks, of which multidisciplinary care was a component. Thus, there was unlikely to be a clear difference in the use of multidisciplinary care within the west of Scotland NHS area after 2000.

\section{Socioeconomic circumstances}

Socioeconomic status was inferred for all patients on the basis of their area of residence at the time of diagnosis, using a deprivation category score, which is a validated seven category ordinal score that ranks all postcode sectors from 1 (most affluent) to 7 (most deprived), using four census variables that have been shown to best correlate with health outcomes: car 
ownership, male unemployment, occupational social classes IV and V (semi-skilled and unskilled occupations, respectively), and overcrowding. ${ }^{28} \mathrm{We}$ grouped scores further into three conventional categories: affluent (scores 1 and 2), intermediate ( 3 to 5), and deprived (6 and 7). ${ }^{14}$ Data from the 1991 census showed that $51 \%$ of people in the intervention area lived in areas classified as most deprived (scores 6 and 7), compared with $18 \%$ of residents in the non-intervention area. ${ }^{29}$

\section{Statistical methods}

We used univariate analyses to examine differences in survival trends between the intervention and non-intervention areas for two time periods, from January 1990 to September 1995 and from October 1995 to December 2000. Where appropriate, we used $\chi^{2}$ and $t$ tests to calculate any significant differences in age, deprivation, and tumour size. If needed, we transformed data to a log scale to satisfy the assumption for the $t$ test.

We defined survival as time from incidence of breast cancer to the earlier of date of death or date of censoring. Patients diagnosed in the earlier period were censored at 30 September 2002 and those diagnosed in the later period were censored at 31 December 2007, to remove any effects of differential follow-up times between the two periods. Deaths due to breast cancer were classified as patients with a main cause of death coded by the Scottish Cancer Registry as ICD-9 codes 174.1 to 174.9 or ICD-10 codes C50.1 to C50.9.

We did an analysis of an interrupted time series to investigate the effect of the intervention while adjusting for trends in survival before the intervention. ${ }^{30}$ Our analysis did not include patients with a date of incidence in 1995, the year the intervention was introduced. We used survival at five year follow-up (after diagnosis) as the outcome measure.

We did multivariable analyses using Cox's proportional hazards models ${ }^{31}$ to examine differences in survival (adjusted for age, deprivation, and year of incidence) between the intervention and non-intervention areas for each time period. The analysis also calculated outcomes in relation to breast cancer mortality and all cause mortality.

We used funnel plots to compare five year survival between hospitals. Funnel plots are control charts in which the event of interest is plotted against a measure of its precision. ${ }^{32}$ Limits for the funnel plots were based on Wilson's formula for binomial confidence intervals. ${ }^{33} \mathrm{We}$ plotted breast cancer survival at five years for each hospital against the number of patients diagnosed with breast cancer in that hospital. To compare survival between hospitals, only one investigator (DSM) knew the code indicating which hospitals comprised the intervention and non-intervention areas. Hospitals with fewer than 25 patients diagnosed in each five year time period were excluded from the funnel plots. This exclusion avoided the range of the y axis scale being too large to distinguish differences between hospitals with larger volumes. We did all analyses using Stata, version 11 software.

\section{Results}

Of 14358 patients reviewed, we excluded 636; 16 patients were diagnosed with inflammatory cancer (ICD for Oncology, 2nd edition, code M8530/3; seven patients in the intervention area, nine in the non-intervention area), and 620 were diagnosed only at death (death certificate only; 182, 438). Of 13722 women in the study population, $6050(44.1 \%)$ were in the intervention area and $7672(55.9 \%)$ in the non-intervention area. Table $1 \Downarrow$ describes patient characteristics.
Age ranged from 18 to 100 years (mean 62.9 years, standard deviation 14.9); $t$ tests of transformed data showed that ages were similar between the intervention and non-intervention areas, both before and after introduction of the intervention. We found a significant difference in deprivation $(\mathrm{P}<0.001)$ between the intervention and non-intervention areas, both before and after the intervention. However, deprivation had not changed significantly in either area over time. In both time periods, more patients in the intervention area than in the non-intervention area lived in the most deprived areas. Data for tumour size was available from 1997 onwards only; available data showed a mean tumour size of $23.9 \mathrm{~mm}$ (standard deviation 14.4); $t$ tests of transformed data showed that tumour size was similar in both the intervention and non-intervention areas.

\section{Survival}

By the end of the follow-up period (seven years from the end of each time period), 4844 (35.3\%) study patients had died of breast cancer and 8301 (60.5\%) had died overall. Breast cancer survival at five years in the time period before introduction of multidisciplinary care was $71.3 \%(\mathrm{n}=2201)$ in the intervention area and $73.6 \%(\mathrm{n}=2881)$ in the non-intervention area. These proportions rose to $79.2 \%(n=2346)$ and $75.9 \%(n=2853)$, respectively, in the time period after the intervention was introduced. Overall survival at five years in the earlier time period was $59.9 \%(\mathrm{n}=1849)$ in the intervention area and $61.5 \%$ $(\mathrm{n}=2408)$ in the non-intervention area. These proportions rose to $65.6 \%(n=1942)$ and $63.8 \%(n=2400)$, respectively, in the later time period.

Our analysis using an interrupted time series was based on breast cancer survival at five years and omitted patients with an incident cancer in 1995 (fig $1 \Downarrow$ ). The analysis showed a significant improvement $(\mathrm{P}=0.004)$ in survival in the intervention area in 1996, compared with the expected survival in that year had the pre-intervention trend continued - that is, had the intervention not been introduced. We saw no corresponding improvement in survival among patients in the non-intervention area $(\mathrm{P}=0.64)$. Overall survival also did not improve in 1996, in either the intervention or non-intervention area. The introduction of the multidisciplinary approach initially had a significant positive impact on breast cancer survival at five years, on incident cancers in 1996, and this impact was maintained (fig 1). We saw a similar pattern in overall survival at five years, but the changes were not significant.

Table $2 \Downarrow$ shows the multivariable survival analyses, using a Cox's proportional hazards model, that was adjusted for age, deprivation, and year of incidence.

\section{Breast cancer mortality}

Before multidisciplinary care was introduced in 1995, breast cancer mortality was higher in the intervention area than in the non-intervention area (hazard ratio $1.11,95 \%$ confidence interval 1.00 to 1.20 ; table 2). After the intervention was introduced, mortality was significantly lower in the intervention area than in the non-intervention area $(0.82,0.74$ to 0.91$)$. Another Cox model showed a significant interaction between time period and intervention area $(\mathrm{P}<0.001)$, indicating that mortality differed significantly between the intervention and non-intervention areas over time. Subgroup analyses by age group showed that the effect of the intervention was strongest in patients aged 80 years and older $(\mathrm{P}=0.001)$, and significant also in patients aged 65-79 years $(\mathrm{P}=0.01)$. We saw no significant effects in either of the younger age groups. 


\section{All cause mortality}

We saw no significant difference in all cause mortality between intervention and non-intervention areas in the earlier time period before multidisciplinary care was introduced (hazard ratio 1.06, $95 \%$ confidence interval 0.98 to 1.14 ; table 2 ). However, in the later time period, mortality was $11 \%$ lower in the intervention area than in the non-intervention area $(0.89,0.82$ to 0.97$)$. As with breast cancer mortality, the effect of the intervention on all cause mortality differed significantly in both the intervention and non-intervention areas $(\mathrm{P}=0.003)$. Subgroup analyses by age group showed a less clear pattern for all cause mortality than that seen for breast cancer mortality. However, the effect of the intervention was most significant in patients aged 80 years or older $(\mathrm{P}=0.002)$; this effect was not seen in any other age group.

\section{Funnel plot analysis}

We used funnel plots to compare breast cancer survival at five years among hospitals before and after the introduction of the intervention in 1995 (fig $2 \Downarrow$ ). A separate funnel plot was created for each time period. In the earlier time period, we plotted breast cancer survival for eight hospitals in the intervention area, and 15 hospitals in the non-intervention area. The number of patients diagnosed per hospital ranged from 30 to 881 in the intervention area and from 26 to 682 in the non-intervention area. Overall mean survival at five year follow-up was $70.3 \%$ (range $36.7-76.8 \%$ in the intervention area, $65.4-86.4 \%$ in the non-intervention area). Three of eight hospitals in the intervention area were below the $95 \%$ confidence limit and two were above. None of the non-intervention hospitals was below the $95 \%$ confidence limit but two were above.

In the later time period, we plotted breast cancer survival for five hospitals in the intervention area, and 10 hospitals in the non-intervention area. The number of patients diagnosed per hospital ranged from 293 to 1025 in the intervention area and from 155 to 775 in the non-intervention area. Overall mean survival at five years during this period $(77.0 \%)$ was higher than that during the earlier time period, with less variation among hospitals (range $78.5-80.2 \%$ in the intervention area, $69.6-80.6 \%$ in the non-intervention area). All hospitals in the intervention area lay within the $95 \%$ confidence limit. One hospital in the non-intervention area was above the $95 \%$ confidence limit and three were below the limit.

\section{Discussion}

\section{Main findings}

We found that the introduction of teams providing multidisciplinary care for the treatment of breast cancer was associated with $18 \%$ lower breast cancer mortality at five years and $11 \%$ lower all cause mortality at five years, compared with similar patients treated in neighbouring areas over the same time period. The introduction of this intervention was also associated with a reduction in the number of hospitals treating breast cancer, reduced variation in survival rates among hospitals, and the reorganisation of breast cancer care away from hospitals with the poorest survival outcomes. Our use of a contemporaneous comparison group suggests that the benefits of multidisciplinary care exceeded those that would otherwise have occurred owing to ongoing improvements in treatment of breast cancer.

The effect size was of a similar magnitude to that found by Gillis and Hole between specialist and non-specialist breast cancer surgeons. ${ }^{34}$ Before the introduction of multidisciplinary teams in the present study, patients with breast cancer in the intervention area were treated by a mixture of specialist and non-specialist surgeons, so it might be expected that only modest improvements in survival would have been achieved by introducing surgery by specialists alone. Although evidence suggests that better local and regional treatment of disease by specialist surgeons explains some of their survival advantage, ${ }^{35}$ the survival advantage of treatment within a multidisciplinary team is unlikely to be explained by surgical specialisation alone. Our results suggest an "empirical induction period" in the intervention area, during which survival continued to increase sharply between 1996 and 1997.

\section{Strengths and weaknesses of the study}

This non-randomised intervention study compared an experimental intervention with no intervention. Although it did not have the strength of a randomised controlled trial, that study design was no longer possible because of the prolific introduction of the multidisciplinary approach of care. The principal limitation of our study was that the observed differences in survival after the introduction of multidisciplinary care in the intervention area might have been due to selection biases or confounding factors.

Although a few patients in our study might have died outside the UK and had been assumed to be alive, we have no reason to believe that this was more likely to occur in the intervention area than the non-intervention area. Selection criteria were applied in the same way to all health board areas-therefore, differential selection of patients with less advanced disease in the intervention area after September 1995 seems unlikely. Indeed, where data for tumour size were available, patients in the intervention area had similar sizes of tumours to those in the non-intervention area.

We excluded all patients with screen detected cancers because they were treated almost exclusively within the intervention area, and such cancers tend to be less advanced and less aggressive. ${ }^{36}$ It is possible that patients treated in the intervention area presented with less advanced disease after September 1995. However, we have no reason to suspect this and available data do not support this explanation.

Women from more affluent areas have consistently been shown to have better survival from breast cancer than those from less affluent areas, ${ }^{37}{ }^{38}$ but the improvement was observed even after adjusting for deprivation. Other concurrent changes in healthcare services, not attributable to the intervention, could have occurred after 1995. However, we are not aware of differences in availability of diagnostic or treatment services between health board areas in the west of Scotland in 1996-2000. No other major interventions, either local or national, were introduced during that time. We are not aware of any formal arrangements to introduce multidisciplinary care in the non-intervention area after 1995, but we do know that trainee surgeons in the intervention area in the early 1990s took up consultant posts in other parts of Scotland. Therefore, improvements in surgical practice could have occurred in the non-intervention area as a result of multidisciplinary working. Such an effect would attenuate rather than increase the true effects of multidisciplinary care, by introducing some misclassification of intervention and non-intervention areas.

\section{Strengths and weaknesses in relation to other studies}

Comparisons have been made between contemporaneous groups of patients given multidisciplinary or conventional treatment, 
within the same geographical area. ${ }^{39}$ However, the multidisciplinary approach probably selects patients with different prognostic factors from the entire patient population, introducing potential selection biases. Patients treated by a multidisciplinary team are also likely to undergo more investigations and be subject to additional clinical audits, so that information about their health and outcomes might differ systematically from those of patients not in multidisciplinary care. These selection and information biases complicate investigations into the true effects of multidisciplinary working. Our study probably did not have these biases, because the intervention was provided for all patients within a geographical area and we obtained the same data from the Scottish Cancer Registry for patients in both intervention and non-intervention areas. We defined the composition and functions of multidisciplinary teams and used the same selection criteria for both areas, thus directly avoiding weaknesses of previous attempts to determine the effects of multidisciplinary care. ${ }^{18}$ Owing to the lack of a precise definition of multidisciplinary care, and the variation in provision of health services in countries outside the UK our results could be difficult to generalise.

\section{Implications for doctors and policy makers}

Multidisciplinary care probably improves patient outcomes by influencing various aspects of care. These factors include adherence to guidelines and nurse education,${ }^{40}$ increased surgical volume and experience, ${ }^{41}$ and improved interdisciplinary working. ${ }^{42}{ }^{43}$ Kingsmore and colleagues reported that the survival benefits of treatment by a specialist surgeon in breast cancer could be explained by better local and regional treatment, as defined by the King's Fund consensus statement. ${ }^{35} 44$ Although multidisciplinary care is considered standard practice in many countries, access to such care still varies. ${ }^{8} 101145$ Our results support the universal provision of cancer care by specialist, multidisciplinary teams. Furthermore, significantly improved survival was achieved among patients aged 80 years and older, which could be an important explanatory element to support the introduction of multidisciplinary care for cancer.

\section{Unanswered questions and future research}

Further work is needed to understand how the multidisciplinary approach affects specific elements of breast cancer care, to further improve the effective management of patients. We found that the benefits of multidisciplinary working were greatest in older patients. Since the intervention guidelines were not age specific, they could have given surgeons and other team members more confidence to actively treat older patients rather than managing them conservatively.

We found that multidisciplinary team care for breast cancer was associated with substantial improvements in survival compared with those we might have expected to occur. The introduction of multidisciplinary care led by specialist breast surgeons performing more than 50 operations per year, resulted in a degree of centralisation of care. Therefore, multidisciplinary teams could increase efficiency by fostering more centralised hospital care. Owing to a lack of data, we were unable to explore the financial cost of multidisciplinary working in our analysis. There is clearly scope for research in this topic; doctors and policy makers alike would benefit from cost effectiveness studies of multidisciplinary working, which could help inform implementation of future programmes. Although multidisciplinary care for cancer has become standard practice in many countries, persisting variations in access to specialist care need to be minimised.
We thank Billy Sloan of the West of Scotland Cancer Surveillance Unit for data linkage; the Information Services Division of NHS National Services Scotland for data collection; and the late David J Hole and Tim $G$ Cooke for their contributions to earlier evaluations of breast cancer outcomes and to the development of the West of Scotland Managed Clinical Network for Breast Cancer.

Contributors: All authors contributed to the design and analysis of the study. EMK wrote the first draft of the manuscript. All authors contributed to the redrafting of the manuscript and approved the final version. EMK is the guarantor. EMK, GMA, and DSM had full access to the data in the study and can take responsibility for the integrity of the data and the accuracy of the data analysis. The other authors had full access to all the results.

Funding: This study was supported by NHS Scotland and University of Glasgow. They had no role in the study design; in the collection, analysis, and interpretation of data; in the writing of the report; and in the decision to submit the article for publication.

Competing interests: All authors have completed the Unified Competing interest form at www.icmje.org/coi_disclosure.pdf (available on request from the corresponding author) and declare: the authors have support from NHS Greater Glasgow and Clyde health board (EMK, GMA), NHS Scotland (HJGB), and the University of Glasgow (WDG, DSM) for the submitted work; no financial relationships with any organisations that might have an interest in the submitted work in the previous 3 years; no other relationships or activities that could appear to have influenced the submitted work.

Ethical approval: The West of Scotland Cancer Surveillance Unit obtained permission to obtain cancer registry data both from Caldicott Guardians of all five health boards in the west of Scotland and from the information services division of the NHS in Scotland privacy advisory committee.

Data sharing: No additional data available.

1 Althuis MD, Dozier JM, Anderson WF, Devesa SS, Brinton LA. Global trends in breast cancer incidence and mortality 1973-1997. Int J Epidemiol April 2005;34:405-12.

2 Chu KC, Tarone RE, Kessler LG, Ries LAG, Hankey BF, Miller BA, et al. Recent trends in US breast cancer incidence, survival, and mortality rates. J Natl Cancer Inst 1996;88:1571-9.

3 Botha JL, Bray F, Sankila R, Parkin DM. Breast cancer incidence and mortality trends in 16 European countries. Eur J Cancer 2003;39:1718-29.

4 Thomson CS, Brewster DH, Dewar JA, Twelves CJ. Improvements in survival for women with breast cancer in Scotland between 1987 and 1993: impact of earlier diagnosis and changes in treatment. Eur $J$ Cancer 2004:40:743-53.

5 Brown SBF, Mallon EA, Edwards J, Campbell FM, McGlynn LM, Elsberger B, et al. Is the biology of breast cancer changing? A study of hormone receptor status 1984-1986 and 1996-1997. Br J Cancer 2009;100:807-10.

$6 \mathrm{Li} \mathrm{Cl}$, Daling JR, Malone KE. Incidence of invasive breast cancer by hormone receptor status from 1992 to 1998. J Clin Oncol 2003;21:28-34.

7 Peto R, Boreham J, Clarke M, Davies C, Beral V. UK and USA breast cancer deaths down by $25 \%$ in year 2000 at ages 20-69 years. Lancet 2000;355:1822.

8 Atun R, Ogawa T, Martin-Moreno JM. Analysis of national cancer control programmes in Europe. 2009. http://spiral.imperial.ac.uk/bitstream/10044/1/4204/1/Cancer\%20Control\% 20vf2.pdf.

9 Stitzenberg K, Meropol N. Trends in centralization of cancer surgery. Ann Surg Oncol 2010;17:2824-31.

10 Anderson BO, Kaufman CS, Keil KD, Carlson RW. Interdisciplinary coordination for breast cancer care: a rational approach to detection, diagnosis, and treatment. Dis Manag Health Outcomes 2008;16:7-11.

11 Department of Health UK. Cancer reform strategy. 2007. www.dh.gov.uk/en/ Publicationsandstatistics/Publications/PublicationsPolicyAndGuidance/DH_081006.

12 Clinical Oncological Society of Australia, Cancer Council Australia, and the National Cancer Control Initiative 2002. Optimising cancer care in Australia. 2003. www. canceraustralia.gov.au/sites/default/files/user-upload/publications/reports/optim_cancer_ care.pdf.

13 Selby P, Gillis C, Haward R. Benefits from specialised cancer care. Lancet 1996;348:313-8.

14 Kingsmore D, Ssemwogerere A, Hole D, Gillis C. Specialisation and breast cancer survival in the screening era. Br J Cancer 2003:88:1708-12.

15 Fosker CJ, Dodwell D. Multidisciplinary team working in cancer: what is the evidence? 2010. www.bmj.com/rapid-response/2011/11/02/cost-mdt.

16 Fleissig A, Jenkins V, Catt S, Fallowfield L. Multidisciplinary teams in cancer care: are they effective in the UK? Lancet Oncol 2006;7:935-43.

17 Taylor C, Munro AJ, Glydodwellnne-Jones R, Griffith C, Trevatt P, Richards M, et al. Multidisciplinary team working in cancer: what is the evidence? BMJ 2010;340:c951.

18 Hong NJL, Wright F, Gagliardi A, Paszat L. Examining the potential relationship between multidisciplinary cancer care and patient survival: an international literature review. J Surg Oncol 2010;102:125-34.

19 Houssami N, Sainsbury R. Breast cancer: multidisciplinary care and clinical outcomes. Eur J Cancer 2006;42:2480. 


\section{What is already known on this topic}

Care by a specialist clinician is associated with improved cancer survival

Treatment of cancer has increasingly been provided within centralised, specialist multidisciplinary teams, but it remains unclear whether such care has contributed to improvements in cancer survival

\section{What this study adds}

Introduction of multidisciplinary care was associated with substantially greater improvements in breast cancer survival over and above those that were expected to occur had the approach not been introduced

Benefits of receiving multidisciplinary care for breast cancer versus conventional care are similar to those of receiving specialist care versus non-specialist care

Introduction of multidisciplinary care was associated with the removal of poorly performing hospitals and reduced variations in outcomes among hospitals.

20 Forrest LM, McMillan DC, McArdle CS, Dunlop DJ. An evaluation of the impact of a multidisciplinary team, in a single centre, on treatment and survival in patients with inoperable non-small-cell lung cancer. Br J Cancer 2005;93:977-8.

21 Stephens MR, Lewis WG, Brewster AE, Lord I, Blackshaw GRJC, Hodzovic I, et al. Multidisciplinary team management is associated with improved outcomes after surgery for esophageal cancer. Dis Eosphagus 2006;19:164-71.

22 NHS Scotland. Introduction of managed clinical networks within the NHS in Scotland. 1999. www.sehd.scot.nhs.uk/mels/1999_10.htm.

23 Kendrick S. The development of record linkage in Scotland: the responsive application of probability matching. In: Committee on Applied and Theoretical Statistics, National Research Council; Federal Committee on Statistical Methodology, Office of Management and Budget. Record linkage techniques-1997: proceedings of an International Workshop and Exposition. National Academies Press, 1999: 319-32. www.nap.edu/catalog.php? record_id=6491.

24 Kendrick SW, Clarke JA. The Scottish medical record linkage system. Health Bull (Edinburgh) 1979;51:72-9.

25 Phipps Al, Ichikawa L, Bowles EJA, Carney PA, Kerlikowske K, Miglioretti DL, et al. Defining menopausal status in epidemiologic studies: a comparison of multiple approaches and their effects on breast cancer rates. Maturitas 2010 9;67:60-6.

26 Laki F, Kirova Y, Savignoni A, Campana F, Levu B, Estve M, et al. Management of operable invasive breast cancer in women over the age of 70: long-term results of a large-scale single-institution experience. Ann Surg Oncol 2010;17:1530-8.

27 Enger SM, Thwin SS, Buist DSM, Field T, Frost F, Geiger AM, et al. Breast cancer treatment of older women in integrated health care settings. J Clin Oncol 2006;24:4377-83.

28 Carstairs V, Morris R. Deprivation and health in Scotland. Aberdeen University Press, 1991.

29 McLaren GB, Bain M. Deprivation and health in Scotland: insights from NHS data. NHS Scotland, 1998

30 Deasy C, Bray JE, Smith K, Wolfe R, Harriss LR, Bernard SA, et al. Cardiac arres outcomes before and after the 2005 resuscitation quidelines implementation: evidence of improvement? Resuscitation 2011;82:984-8.

31 Cox DR. Regression models and life tables. J R Stat Soc (B) 1972;34:187-220.

32 Spiegelhalter DJ. Handling over-dispersion of performance indicators. Qual Saf Health Care 2005;14:347-51

33 Newcombe RG. Two-sided confidence intervals for the single proportion: comparison of seven methods. Stat Med 1998:17:857-72.

34 Gillis CR, Hole DJ. Survival outcome of care by specialist surgeons in breast cancer: a study of 3786 patients in the west of Scotland. BMJ 1996;312:145-8.
35 Kingsmore D, Hole D, Gillis C. Why does specialist treatment of breast cancer improve survival? The role of surgical management. Br J Cancer 2004;90:1920.

36 Weaver DL, Rosenberg RD, Barlow WE, Ichikawa L, Carney PA, Kerlikowske K, et al. Pathologic findings from the Breast Cancer Surveillance Consortium. Cancer 2006;106:732-42.

37 Thomson CS, Hole DJ, Twelves CJ, Brewster DH, Black RJ. Prognostic factors in women with breast cancer: distribution by socioeconomic status and effect on differences in survival. J Epidemiol Community Health 2001;55:308-15

38 Schrijvers CTM, Mackenbach JP, Lutz J-M, Quinn MJ, Coleman MP. Deprivation and survival from breast cancer. Br J Cancer 1995;72:738-43.

39 Friedland PL, Bozic B, Dewar J, Kuan R, Meyer C, Phillips M. Impact of multidisciplinary team management in head and neck cancer patients. Br J Cancer 2011;104:1246-8.

40 McCarthy M, Datta P, Khachatryan A, Coleman MP, Rachet B. Would compliance with cancer care standards improve survival for breast, colorectal and lung cancers? J Epidemiol Community Health 2008;62:650-4.

41 Bilimoria KY, Bentrem DJ, Feinglass JM, Stewart AK, Winchester DP, Talamonti MS, et al. Directing surgical quality improvement initiatives: comparison of perioperative mortality and long-term survival for cancer surgery. J Clin Oncol 2008;26:4626-33.

42 Junor EJ, Hole DJ, Gillis CR. Management of ovarian cancer: referral to a multidisciplinary team matters. Br J Cancer 1994;70:363-70.

43 Ruhstaller T, Roe H, Thürlimann B, Nicoll JJ. The multidisciplinary meeting: an indispensable aid to communication between different specialities. Eur $\mathrm{J}$ Cancer 2006;42:2459-62.

44 King's Fund Forum Consensus Statement. Consensus development conference: treatment of primary breast cancer. BMJ 1986;293:946-7.

45 Saini KS, Taylor C, Ramirez AJ, Palmieri C, Gunnarsson U, Schmoll HJ, et al. Role of the multidisciplinary team in breast cancer management: results from a large international survey involving 39 countries. Ann Oncol 2012;23:853-9.

\section{Accepted: 16 March 2012}

\section{Cite this as: BMJ 2012;344:e2718}

This is an open-access article distributed under the terms of the Creative Commons Attribution Non-commercial License, which permits use, distribution, and reproduction in any medium, provided the original work is properly cited, the use is non commercial and is otherwise in compliance with the license. See: http://creativecommons.org/licenses/by$\mathrm{nc} / 2.0 /$ and http://creativecommons.org/licenses/by-nc/2.0/legalcode. 


\section{Tables}

Table 1| Age and socioeconomic characteristics of 13<thin>722 female patients with breast cancer in the west of Scotland, 1990-2000. Data are no (\%)

\begin{tabular}{|c|c|c|c|c|}
\hline & \multicolumn{2}{|c|}{ January 1990 to September 1995} & \multicolumn{2}{|c|}{ October 1995 to December 2000} \\
\hline & $\begin{array}{c}\text { Non-intervention group } \\
\quad(n=3913)\end{array}$ & Intervention group $(\mathrm{n}=3088)$ & Non-intervention group $(n=3759)$ & Intervention group $(\mathrm{n}=2962)$ \\
\hline \multicolumn{5}{|l|}{ Age (years) } \\
\hline$\leq 49$ & $853(21.8)$ & $735(23.8)$ & $823(21.9)$ & $739(24.9)$ \\
\hline $50-64$ & $1299(33.2)$ & $996(32.3)$ & $1131(30.1)$ & $751(25.4)$ \\
\hline $65-79$ & $1236(31.6)$ & $948(30.7)$ & $1220(32.5)$ & $1032(34.8)$ \\
\hline$\geq 80$ & $525(13.4)$ & $409(13.2)$ & $585(15.6)$ & $440(14.9)$ \\
\hline \multicolumn{5}{|c|}{ Deprivation category } \\
\hline Most affluent & $448(11.4)$ & $548(17.7)$ & 449 (11.9) & $533(18.0)$ \\
\hline Intermediate & 2745 (70.2) & $1298(42.0)$ & $2684(71.4)$ & $1255(42.4)$ \\
\hline Most deprived & $720(18.4)$ & $1242(40.2)$ & $626(16.7)$ & $1174(39.6)$ \\
\hline \multicolumn{5}{|c|}{ Tumour size (mm) } \\
\hline$\leq 19$ & - & - & $1090(54.2)^{*}$ & $764(52.3)^{*}$ \\
\hline $20-39$ & - & - & $760(37.8)^{*}$ & $543(37.2)^{*}$ \\
\hline$\geq 40$ & - & - & $161(8.0)^{*}$ & $153(10.5)^{*}$ \\
\hline Not recorded & $3913(100)$ & $3088(100)$ & $1748(46.5)$ & $1502(50.7)$ \\
\hline
\end{tabular}

*Percentages calculated using number of patients with available data as the denominator. 


\begin{tabular}{|c|c|c|c|c|c|}
\hline \multirow[b]{2}{*}{ Patient age group and time period of analysis } & \multirow[b]{2}{*}{$\mathbf{N}$} & \multicolumn{2}{|c|}{ Breast cancer specific mortality } & \multicolumn{2}{|c|}{ All cause mortality } \\
\hline & & Hazard ratio $(95 \% \mathrm{Cl})^{\star}$ & $\mathbf{P}$ & Hazard ratio $(95 \% \mathrm{CI})^{\star}$ & $\mathbf{P}$ \\
\hline \multicolumn{6}{|l|}{ All cases } \\
\hline January 1990 to September 1995 & 7001 & $1.11(1.00$ to 1.20$)$ & 0.04 & $1.06(0.98$ to 1.14$)$ & 0.13 \\
\hline October 1995 to December 2000 & 6721 & $0.82(0.74$ to 0.91$)$ & $<0.001$ & $0.89(0.82$ to 0.97$)$ & 0.005 \\
\hline Interaction between time period and area & 13722 & - & $<0.001$ & - & 0.003 \\
\hline \multicolumn{6}{|l|}{ Age $\leq 49$ years } \\
\hline January 1990 to September 1995 & 1588 & $1.05(0.86$ to 1.29$)$ & 0.63 & $1.03(0.85$ to 1.25$)$ & 0.77 \\
\hline October 1995 to December 2000 & 1562 & $1.04(0.81$ to 1.32$)$ & 0.78 & $1.13(0.90$ to 1.42$)$ & 0.31 \\
\hline Interaction between time period and area & 3150 & - & 0.95 & - & 0.52 \\
\hline \multicolumn{6}{|l|}{ Age $50-64$ years } \\
\hline January 1990 to September 1995 & 2295 & $1.14(0.96$ to 1.34$)$ & 0.13 & $1.18(1.01$ to 1.37$)$ & 0.03 \\
\hline October 1995 to December 2000 & 1882 & $0.92(0.74$ to 1.13$)$ & 0.42 & $0.94(0.78$ to 1.14$)$ & 0.55 \\
\hline Interaction between time period and area & 4177 & - & 0.11 & - & 0.07 \\
\hline \multicolumn{6}{|l|}{ Age $65-79$ years } \\
\hline January 1990 to September 1995 & 2184 & $1(0.85$ to 1.18$)$ & 0.97 & $0.95(0.83$ to 1.08$)$ & 0.44 \\
\hline October 1995 to December 2000 & 2252 & $0.72(0.60$ to 0.85$)$ & $<0.001$ & $0.87(0.77$ to 1.00$)$ & 0.05 \\
\hline Interaction between time period and area & 4436 & - & 0.01 & - & 0.47 \\
\hline \multicolumn{6}{|l|}{ Age $\geq 80$ years } \\
\hline January 1990 to September 1995 & 934 & $1.24(1.00$ to 1.56$)$ & 0.05 & $1.08(0.93$ to 1.26$)$ & 0.33 \\
\hline October 1995 to December 2000 & 1025 & $0.75(0.59$ to 0.94$)$ & 0.01 & $0.78(0.67$ to 0.91$)$ & 0.002 \\
\hline Interaction between time period and area* & 1959 & - & 0.001 & - & 0.002 \\
\hline
\end{tabular}

${ }^{*}$ Adjusted for year of incidence, deprivation, and (where appropriate) age. 


\section{Figures}
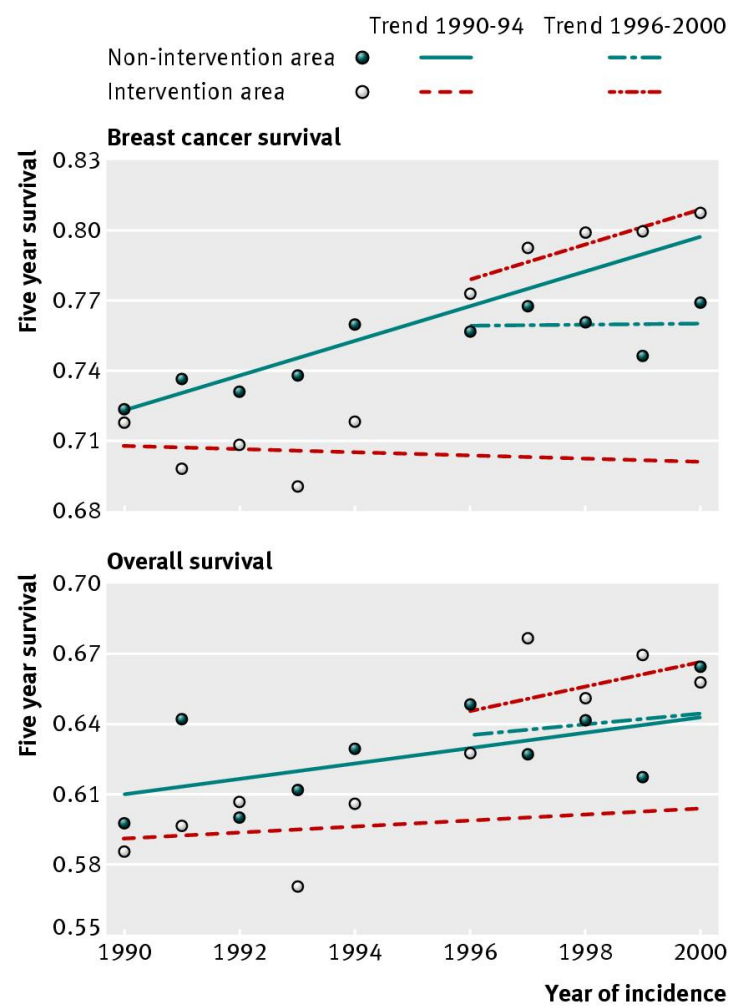

Fig 1 Five year survival by year of incidence with trends before and after year 1995, by intervention area
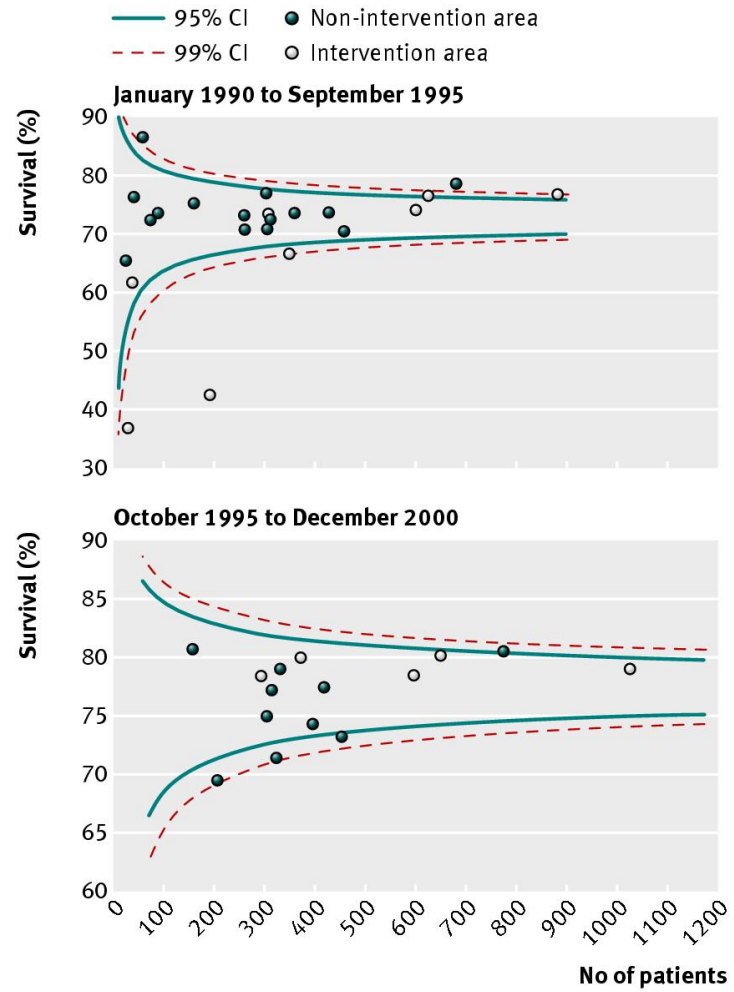

Fig 2 Funnel plot of breast cancer survival at five years in study hospitals, by time period. Hospitals diagnosing fewer than 25 patients in each time period were excluded (of which more than $75 \%$ diagnosed fewer than three cases of breast cancer) 